\title{
Optimized Design of a Converter Decimal to BCD Encoder and a Reversible 4-bit Controlled Up/Down Synchronous Counter
}

\author{
Issam Andaloussi ${ }^{1}$ \\ Faculty of Sciences Physics, \\ Physics Department \\ Ibn Tofail University, \\ Kénitra, Morocco
}

\author{
Sedra Moulay Brahim ${ }^{2}$ \\ Faculty of Sciences and Techniques, \\ Moulay Ismail University, \\ BP509, Boutalamine 52000, \\ Errachidia, Morocco
}

\author{
Mariam El Ghazi ${ }^{3}$ \\ Faculty of Sciences \\ Computer Sciences Department \\ Abdelmalek Essaadi University, \\ Tetouan, Morocco
}

\begin{abstract}
The field of quantum computing, reversible logic, and nanotechnology have earned much attention from researchers in recent years due to their low power dissipation. Quantum computing has been a guiding light for nanotechnology, optical computing of information, low power CMOS design, computer science. Moreover, the dissipation of energy in the field combinatorial logic circuits becomes one of the most important aspects to be avoided. This problem is remedied by a reversible logic favoring the reproduction of inputs to outputs, which is due to the absence of unused bits. Every bit of information not used generates a loss of information causing a loss of energy under the form of heat, the reversible logic leads to zero heat dissipation. Among the components affected by reversible logic are binary reversible counter and converter from decimal to $B C D$ encoder(D2BE) which are considered essential elements. This article will propose an optimized reversible design of a converter from decimal to BCD encoder (D2BE) and an optimized design of reversible Binary counter with up/ down. Our designs show an improvement compared to previous works by replacing some reversible gates with others while keeping the same functionality and improving performance criteria in terms of the number of gates, garbage outputs, constant inputs, quantum cost, delay, and Hardware complexity.
\end{abstract}

Keywords-Decimal to BCD Encoder (D2BE); Reversible Binary Counter; Number of Gates (CG); Number of Garbage Output (NGO); Number of Constant Inputs (NCI); Quantum Cost (QC); Hardware Complexity (HC)

\section{INTRODUCTION}

In the irreversible logic, the design of circuits becomes more and more difficult in terms of material design, this is due to the dissipation of energy in the form of heat which is generated at the end of the lost or unused bits. In irreversible logical calculation, each unused bit generates a loss of energy which is expressed by the formula KTln2 or K: constant of BOLTZMAN and T: absolute temperature related to calculation, this formula was established by Landauer in 1960 [1]. In digital circuits, energy loss is a function of garbage outputs (unused bits). Bennett has proven that this loss of energy can be avoided by a reversible logic using reversible gates [2]. Toffoli [3] shown in 1980 that to avoid a zero internal power circuit, it can be designed using reversible logic gates which, inside each of them, have a certain bijectivity [4-6] between inputs and outputs. Each reversible gate must have a tie between the number of inputs and that of outputs [4-6] and each input vector can be uniquely deduced from the output vector [4-6]. Performance criteria to be improved and concerned by this study are: Number of gates (CG), Number of garbage outputs (NGO) The number of constant inputs (NCI) and Quantum cost (QC). Fredkin and Toffoli have shown that the more NCI and NGO are minimized, the more efficient the circuit design is improved [7]. It was shown in [8-10] that the decrease in energy dissipation in the form of heat is proportional to the minimization of the NGO which shows the great importance of these criteria.

Our work will be divided into two parts the first concerns the design of a converter from decimal to BCD encoder based on a recent study design6 [14]: to modify it and improve the performance criteria compared to the latter and even to the five other studies design5 [14], design4 [13], design3 [12], design2 [12] and design1 [11]. The second concerns the design of a reversible binary counter based on a recent study design 3 [17]by modifying it while keeping its same functionality and increasing the performance criteria compared to the latter and even to the two other studies design2 [16] and design1 [15].

The rest of this article is organized as follows: the second section presents the reversible gates concerned by this article by showing their performance criteria, namely the quantum cost that is deducted from the associated quantum implementation, and the hardware complexity. The third section presents a literature review of the recent designs proposed related to each study the design of the converter from decimal to BCD encoder and the reversible binary counter while showing the performance criteria of each, for the first study we have six proposals designs and for the second one we have three.

The fourth section shows our proposed designs of the D2BE circuit and reversible binary counter. The fifth section will show the result and discussion of our work by revealing our proposed designs and calculate our performance criteria while showing the percentages of improvement obtained.

Finally, a conclusion and perspectives in the last section.

\section{The Reversible Gates Concerned by the Study AND THEIR PERFORMANCE CRITERIA}

In this section we will define the six performance criteria concerned by this article which are: 


\section{A. Performance Criteria}

1) Number of Gates (CG): The number of gates required to make a circuit [18].

2) Number of garbage outputs (NGO): The unused or unwanted logic outputs of the reversible gate maintain in the output lines to make the circuit reversible [22].

3) Number of Constant Inputs (NCI): The number of inputs must remain constant at 0 or 1 to integrate the given logic function [6].

4) Quantum Cost $(Q C)$ : The QC is calculated by counting the number of one input-output and two input-output reversible gates used in realizing a circuit $[19,20]$. The QC of one input-output and two input-output reversible gates is realized to be 1 .

5) Hardware Complexity $(\mathrm{HC})$ : The number of fundamental operations (Ex-OR, AND, NO, etc.) required to make the circuit. Actually, a constant complexity is supposed for each fundamental operation of the circuit, such as $\alpha$ for Ex-OR, $\beta$ for AND, $\delta$ for NOT, etc. Eventually, the entire number of operations is calculated in terms of $\alpha, \beta$, and $\delta$ [23].

In this part, we will present the reversible gates that we will use in this article by showing their performance criteria, specially the quantum cost that we deduce directly from the quantum implementation, and its hardware complexity.

\section{B. Feyman Gate FG}

A reversible gate $2 * 2$ have as inputs $\mathrm{A}$ and $\mathrm{B}$ and as outputs $\mathrm{P}=\mathrm{A}$ and $\mathrm{Q}=\mathrm{A} \bigoplus \mathrm{B}$. The quantum cost of the gate $\mathrm{FG}$ is worth $\mathrm{QC}=1$, its Hardware complexity is worth $\mathrm{HC}=$ $1 \alpha[18]$.

\section{TS-3 Gate}

A reversible gate $3 * 3$ have for inputs $\mathrm{A}, \mathrm{B}$ and $\mathrm{C}$ and as outputs $\mathrm{P}=\mathrm{A}, \mathrm{Q}=\mathrm{B}$ and $\mathrm{R}=\mathrm{A} \oplus \mathrm{B}$. The quantum cost of the TS-3 gate is equal to $\mathrm{QC}=2$, its Hardware complexity is worth $\mathrm{HC}=2 \alpha$ [24].

\section{Fredkin Gate FRG}

A reversible gate $3 * 3$ have as inputs $\mathrm{A}, \mathrm{B}$ and $\mathrm{C}$ and as outputs $\mathrm{P}=\mathrm{A}, \mathrm{Q}=\mathrm{A}^{\prime} \mathrm{B} \bigoplus \mathrm{AC}$ and $\mathrm{R}=\mathrm{A}^{\prime} \mathrm{C} \bigoplus \mathrm{AB}$. The quantum cost of the FRG gate is equal to $\mathrm{QC}=2$, its Hardware complexity is equal to $\mathrm{HC}=2 \alpha+4 \beta+1 \delta$ [18].

\section{E. Peres Gate PG}

A reversible gate $3 * 3$ have as inputs $A, B$ and $C$ and as outputs $\mathrm{P}=\mathrm{A}, \mathrm{Q}=\mathrm{A} \bigoplus$ and $\mathrm{R}=\mathrm{AB} \bigoplus \mathrm{C}$. The quantum cost of the gate $\mathrm{PG}$ is equal to $\mathrm{QC}=4$, its Hardware complexity is worth $\mathrm{HC}=2 \alpha+1 \beta$ [25].

\section{F. HNFG Gate}

A reversible gate $4 * 4$ have for inputs $A, B, C$ and $D$ as outputs $\mathrm{P}=\mathrm{A}, \mathrm{Q}=\mathrm{A} \bigoplus \mathrm{C}, \mathrm{R}=\mathrm{B}$ and $\mathrm{S}=\mathrm{B} \bigoplus \mathrm{D}$. The quantum cost of the HNFG gate is equal to $\mathrm{QC}=4$, its Hardware complexity is worth $\mathrm{HC}=2 \alpha$ [18].

\section{G. HNG Gate}

A reversible gate $4 * 4$ have as inputs $\mathrm{A}, \mathrm{B}, \mathrm{C}$ and $\mathrm{D}$ as outputs $\mathrm{P}=\mathrm{A}, \mathrm{Q}=\mathrm{B}, \mathrm{R}=\mathrm{A} \bigoplus \mathrm{B} \oplus \mathrm{C}$ and $\mathrm{S}=(\mathrm{A} \oplus \mathrm{B})$ $\mathrm{C} \bigoplus \mathrm{AB} \bigoplus \mathrm{D}$. The quantum cost of the HNG gate is equal to $\mathrm{QC}=6$, its Hardware complexity is worth $\mathrm{HC}=4 \alpha+2$ $\beta[18]$.

\section{H. RSJ Gate}

A reversible gate $4 * 4$ have as inputs $\mathrm{A}, \mathrm{B}, \mathrm{C}$ and $\mathrm{D}$ as outputs $\mathrm{P}=\mathrm{A}, \mathrm{Q}=\mathrm{B}, \mathrm{R}=\mathrm{AB} \bigoplus \mathrm{C}, \mathrm{S}=\mathrm{AB} \bigoplus \mathrm{D}$. The quantum cost of the RSJ gate is equal to $\mathrm{QC}=12$ and its Hardware complexity is equal to $\mathrm{HC}=2 \alpha+1 \beta$ [26].

\section{TFG Gate}

A reversible gate $4 * 4$ have as inputs $\mathrm{A}, \mathrm{B}, \mathrm{C}$ and $\mathrm{D}$ as outputs $\mathrm{P}=\mathrm{A}, \mathrm{Q}=\mathrm{A} \oplus \mathrm{B}, \mathrm{R}=\mathrm{AB} \oplus \mathrm{C}, \mathrm{S}=\mathrm{AB} \oplus \mathrm{C}$ bigoplus $\mathrm{D}$. The quantum cost of the TFG gate is equal to $\mathrm{QC}$ $=5$ and its Hardware complexity is equal to $\mathrm{HC}=3 \alpha+1 \beta$ [15].

\section{J. TKS Gate}

A reversible gate $4 * 4$ have as inputs $\mathrm{A}, \mathrm{B}$, and $\mathrm{C}$ as outputs $\mathrm{P}=\mathrm{AC}$ ' $+\mathrm{BC}, \mathrm{Q}=\mathrm{A} \bigoplus \mathrm{B} \bigoplus \mathrm{C}, \mathrm{R}=\mathrm{AC}+\mathrm{BC}$ '. The quantum cost and the Hardware complexity of the TKS gate is not mentioned [27].

\section{K. MSH Gate}

A reversible gate $4 * 4$ have as inputs $\mathrm{A}, \mathrm{B}, \mathrm{C}$ and $\mathrm{D}$ as outputs $\mathrm{P}=\mathrm{A}, \mathrm{Q}=\mathrm{B} \bigoplus \mathrm{C}, \mathrm{R}=\mathrm{A}^{\prime} \mathrm{C} \bigoplus \mathrm{AB}$ and $\mathrm{S}=\mathrm{A}^{\prime} \mathrm{C} \bigoplus$ $\mathrm{AB} \oplus \mathrm{D}$. The quantum cost of the $\mathrm{MSH}$ gate is equal to $\mathrm{QC}$ $=6$ and its Hardware complexity is equal to $\mathrm{HC}=3 \alpha+2 \beta$ $+1 \delta[28]$.

\section{NP Gate}

A reversible gate $4 * 4$ have as inputs $\mathrm{A}, \mathrm{B}, \mathrm{C}$ and $\mathrm{D}$ as outputs $\mathrm{P}=\mathrm{A}, \mathrm{Q}=\mathrm{A}^{\prime} \mathrm{B} \bigoplus \mathrm{AC} \mathrm{C}^{\prime}, \mathrm{R}=\mathrm{A}^{\prime} \mathrm{C} \bigoplus \mathrm{AB}$ and $\mathrm{S}=$ $\mathrm{A}^{\prime} \mathrm{C} \bigoplus \mathrm{AB} \bigoplus \mathrm{D}$. The quantum cost of the gate NP is equal to $\mathrm{QC}=5$ and its Hardware complexity is equal to $\mathrm{HC}=3 \alpha$ $+4 \beta+2 \delta$ [29].

\section{BJN Gate}

A reversible gate $3 * 3$ have as inputs $A, B$ and $D$ as outputs $\mathrm{P}=\mathrm{A}, \mathrm{Q}=\mathrm{B}, \mathrm{R}=(\mathrm{A}+\mathrm{B}) \bigoplus \mathrm{C}$. The quantum cost of the gate $\mathrm{BJN}$ is equal to $\mathrm{QC}=5$ and its Hardware complexity is $\mathrm{HC}=3 \alpha+4 \beta+2 \delta[30]$.

\section{N. Sayem Gate SG}

A reversible gate $4 * 4$ have as inputs $\mathrm{A}, \mathrm{B}, \mathrm{C}$ and $\mathrm{D}$ as outputs $\mathrm{P}=\mathrm{A}, \mathrm{Q}=\mathrm{A}^{\prime} \mathrm{B} \bigoplus \mathrm{AC}, \mathrm{R}=\mathrm{A}^{\prime} \mathrm{B} \bigoplus \mathrm{AC} \bigoplus \mathrm{D}$ and $\mathrm{S}=\mathrm{AB} \oplus \mathrm{A}^{\prime} \mathrm{C} \oplus \mathrm{D}$. The quantum cost of the gate $\mathrm{SG}$ is equal to $\mathrm{QC}=5$ and its Hardware complexity is equal to $\mathrm{HC}$ $=4 \alpha+4 \beta+1 \delta[31]$.

\section{LiterATURE REVIEW}

We will present all the recent studies related to our proposed designs, starting by: 


\section{A. Design of the Converter from Decimal to BCD Encoder or $D 2 B E$}

The decimal number system is made up of ten numbers from zero to nine to convert it in BCD Format, using a system called D2BE composed of ten inputs and four outputs. the inputs range from D0 to D9 and the outputs are $\mathrm{A}, \mathrm{B}, \mathrm{C}$, and $D$ [11] [12] [21] outputs are expressed depending on the inputs as follows:

$\mathrm{A}=\mathrm{D} 8 \bigoplus \mathrm{D} 9$

$\mathrm{B}=\mathrm{D} 4 \oplus \mathrm{D} 5 \oplus \mathrm{D} 6 \oplus \mathrm{D} 7$

$\mathrm{C}=\mathrm{D} 2 \oplus \mathrm{D} 3 \oplus \mathrm{D} 6 \oplus \mathrm{D} 7$

$\mathrm{D}=\mathrm{D} 1 \oplus \mathrm{D} 3 \oplus \mathrm{D} 5 \oplus \mathrm{D} 7 \oplus \mathrm{D} 9$

Table I presents the truth table of the converter from decimal to $\mathrm{BCD}$ encoder.

TABLE I. D2BE CONVERTER TRUTH TABLE

\begin{tabular}{|c|c|c|c|c|c|c|c|c|c||c|c|c|c|}
\hline D9 & D8 & D7 & D6 & D5 & D4 & D3 & D2 & D1 & D0 & A & B & C & D \\
\hline 0 & 0 & 0 & 0 & 0 & 0 & 0 & 0 & 0 & 1 & 0 & 0 & 0 & 0 \\
\hline 0 & 0 & 0 & 0 & 0 & 0 & 0 & 0 & 1 & 0 & 0 & 0 & 0 & 1 \\
\hline 0 & 0 & 0 & 0 & 0 & 0 & 0 & 1 & 0 & 0 & 0 & 0 & 1 & 0 \\
\hline 0 & 0 & 0 & 0 & 0 & 0 & 1 & 0 & 0 & 0 & 0 & 0 & 1 & 1 \\
\hline 0 & 0 & 0 & 0 & 0 & 1 & 0 & 0 & 0 & 0 & 0 & 1 & 0 & 0 \\
\hline 0 & 0 & 0 & 0 & 1 & 0 & 0 & 0 & 0 & 0 & 0 & 1 & 0 & 1 \\
\hline 0 & 0 & 0 & 1 & 0 & 0 & 0 & 0 & 0 & 0 & 0 & 1 & 1 & 0 \\
\hline 0 & 0 & 1 & 0 & 0 & 0 & 0 & 0 & 0 & 0 & 0 & 1 & 1 & 1 \\
\hline 0 & 1 & 0 & 0 & 0 & 0 & 0 & 0 & 0 & 0 & 1 & 0 & 0 & 0 \\
\hline 1 & 0 & 0 & 0 & 0 & 0 & 0 & 0 & 0 & 0 & 1 & 0 & 0 & 1 \\
\hline
\end{tabular}

The inputs of the converter from decimal to $\mathrm{BCD}$ encoder are: D9, D8, D7, D6, D5, D4, D3, D2, D1 and D0 and its outputs are:A,B,C and D.

We present below the related recent studies:

1) Design1: In 2012 JUN-CHAO WANGI [11] designed the converter from decimal to BCD encoder composed of five CNOT gates and fifteen Toffoli Gate gates (Fig. 1). According to the circuit proposed by JUN-CHAO WANGI, we have the performance criteria as follows:

-Number of gates: Its proposed circuit consists of five CNOT gates and 15 Toffoli gates. therefore $\mathrm{CG}=20$.

-Number of garbage outputs: As it indicated $\mathrm{NGO}=25$.

-Number of constant inputs: This criteria is not mentioned.

-Quantum cost: Its circuit is composed of five CNOT reversible gates having $\mathrm{QC}=5(\mathrm{QC}=1$ for each $)$ and fifteen TG reversible gates having $\mathrm{QC}=75(\mathrm{QC}=5$ for each) therefore $\mathrm{QC}=80$.

-Hardware complexity: The circuit contains five CNOT reversible gates and fifteen TG reversible gates so $\mathrm{HC}=5 \alpha$ $+15(1 \alpha+1 \beta)=20 \alpha+15 \beta$.

2) Design2 and Design3: In 2014 VANDANA SHUKLA [12] designed 2 designs of the decimal to $\mathrm{BCD}$ converter encoder which are as follows:

*Design2: This design is the circuit of the converter from decimal to BCD encoder design2 as shown in Fig. 2. According to this proposed circuit we have the performance criteria as follows:

-Number of gates: Its proposed circuit consists of 12 FG gates including four gates reversible TKS therefore $\mathrm{CG}=16$.

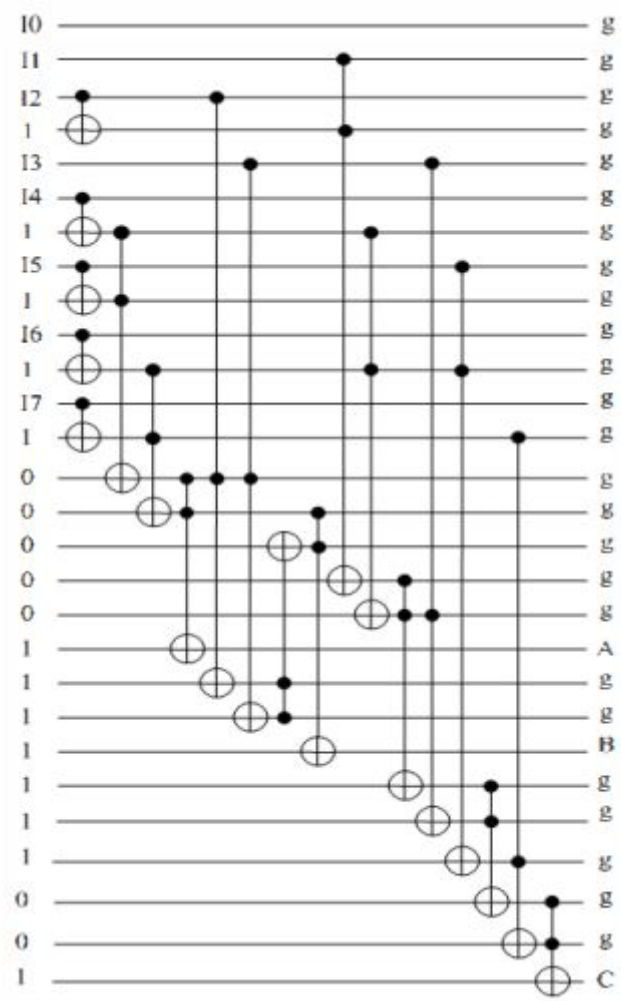

Fig. 1. Design1 D2BE.

-Number of garbage outputs: Proposed circuit consists of 12 FG gates including seven among they have one garbage output and four TKS reversible gates, each of which has two garbage outputs so $\mathrm{NGO}=15$.

-Number of constant inputs: Proposed circuit consists of 12 FG gates including two among them one constant entrance and four TKS reversible gates, each of which has one entrance constant therefore $\mathrm{NCI}=6$.

-Quantum cost: The quantum cost is not mentioned by VANDANA SHUKLA.

-Hardware complexity: The circuit contains 12 reversible gates FG and four TKS reversible gates so $\mathrm{HC}=12 \alpha+4$ ( $2 \alpha$ $+4 \beta+1 \delta)=20 \alpha+16 \beta+4 \delta$.

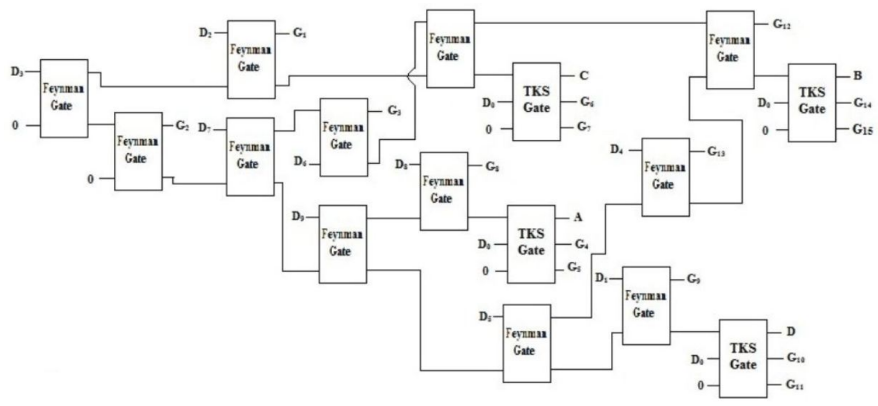

Fig. 2. Design2 D2BE. 
*Design3: This design presents the decimal to $\mathrm{BCD}$ converter circuit design3 encoder (Fig. 3). According to this proposed circuit we have the performance criteria as follows: -Number of gates: Its proposed circuit consists of 10 reversible $\mathrm{BJN}$ gates therefore $\mathrm{CG}=10$.

-Number of garbage outputs: Its proposed circuit consists of $10 \mathrm{BJN}$ reversible gates of which six among them have two garbage output and three of them have one garbage output and one reversible gate $\mathrm{BJN}$ has no garbage output so $\mathrm{NGO}=15$.

-Number of constant inputs: The proposed circuit consists of 10 reversible gates BJN each of which has one constant input so $\mathrm{NCI}=10$.

-Quantum cost: The proposed circuit consists of $10 \mathrm{BJN}$ reversible gates having $\mathrm{QC}=50$ (including $\mathrm{QC}=5$ for each) so $\mathrm{QC}=50$.

-Hardware complexity: The circuit contains 10 reversible gates $\mathrm{BJN}$ so $\mathrm{HC}=10 \alpha$.

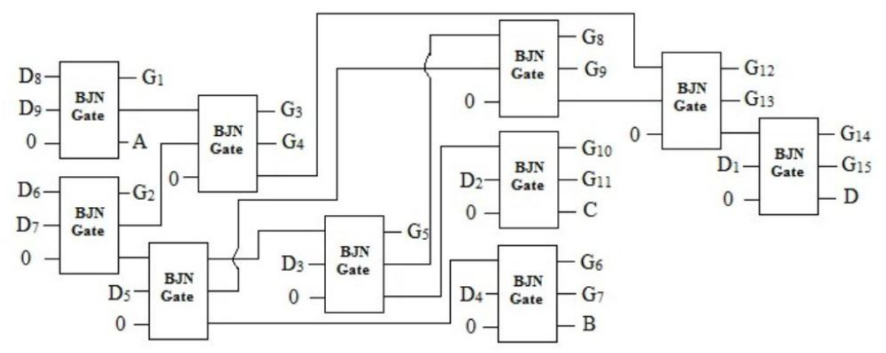

Fig. 3. Design3 D2BE.

3) Design4: In 2016 KUNAL CHAUDHARY [13] devised a design for the converter from decimal to $\mathrm{BCD}$ encoder is composed of four reversible gates Fredkin Gate FRG and three reversible gates FG in the following figure (Fig. 4). According to this proposed circuit we have the performance criteria as follows:

-Number of gates: $\mathrm{CG}=11$.

-Number of garbges outputs: $\mathrm{NGO}=11$.

-Number of constant inputs: $\mathrm{NCI}=5$.

-Quantum cost: $\mathrm{QC}=23$.

-Hardware complexity:The circuit contains 10 reversible gates Fredkin gate so $\mathrm{HC}=4(2 \alpha+4 \beta+1 \delta)+3 \alpha \mathrm{HC}=11 \alpha+16$ $\beta+7 \delta$.

4) Design5 et Design6: In 2019 SHEBA DIAMOND THABAH [14] devised two designs of the decimal converter to $\mathrm{BCD}$ encoder which are as follows:

*Design5: The decimal to BCD converter circuit design5 encoder as explained in Fig. 5. According to this proposed circuit we have the performance criteria as follows:

-Number of gates: Its proposed circuit consists of 10 reversible PG gates, of which therefore $\mathrm{CG}=10$.

-Number of garbage outputs: Its proposed circuit consists of 10 reversible PG gates of which five of them have two garbage output and five of them have one garbage output so $\mathrm{NGO}=15$

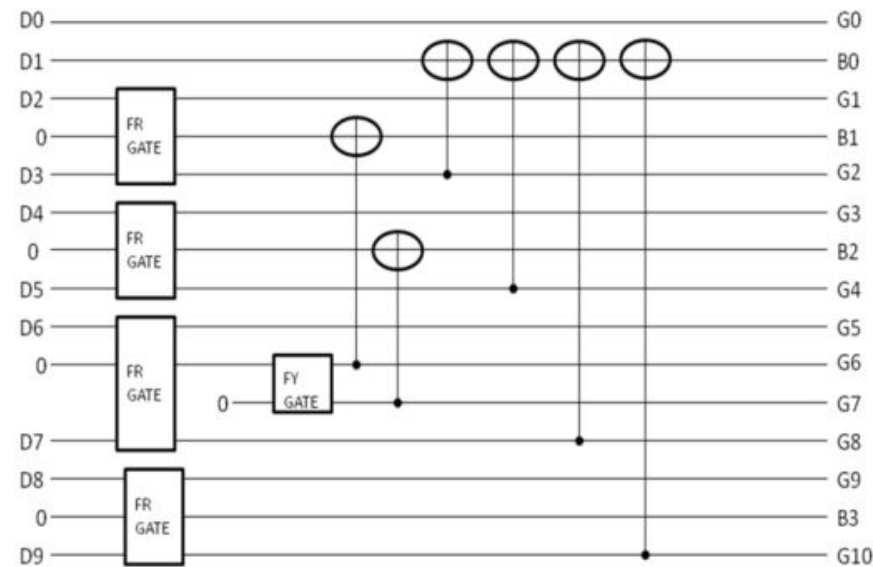

Fig. 4. Design4 D2BE.

-Number of constant inputs: Proposed circuit consists of 10 reversible PG gates of which nine of them has one constant input and the tenth has no constant input therefore $\mathrm{NCI}=9$.

- Quantum cost: Its proposed circuit consists of 10 reversible $\mathrm{PG}$ gates having $\mathrm{QC}=40$ (including $\mathrm{QC}=4$ for each) so $\mathrm{QC}=40$.

-Hardware complexity: The circuit contains 10 reversible gates Peres gate so: $\mathrm{HC}=10(2 \alpha+1 \beta) \mathrm{HC}=20 \alpha+10 \beta$

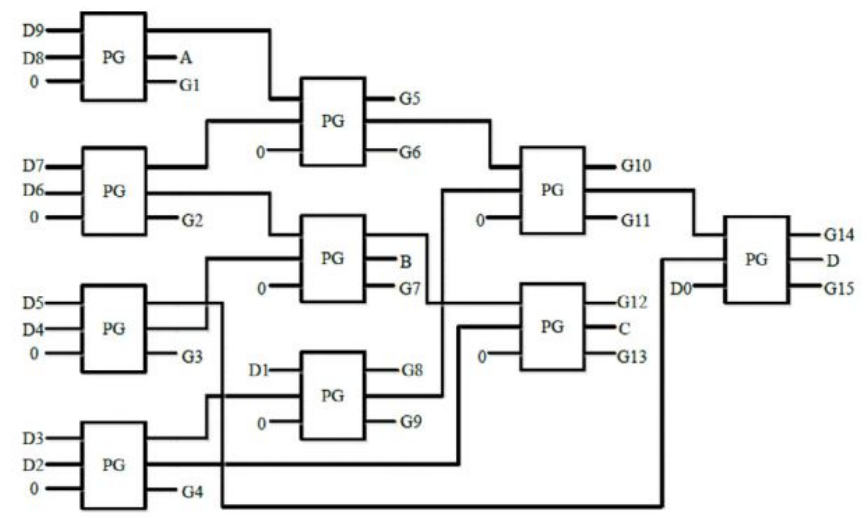

Fig. 5. Design5 D2BE.

*Design6: The decimal to BCD converter circuit design6 encoder (Fig. 6). According to this proposed circuit, we have the performance criteria as follows:

-Number of gates: Its proposed circuit consists of 11 FG reversible gates therefore $\mathrm{CG}=11$.

-Number of garbage outputs: Its proposed circuit consists of 10 FG reversible gates of which five of them have one garbage output and one of them has two garbage outputs and five among them has no garbage output so $\mathrm{CG}=7$.

-Number of constant inputs: Its proposed circuit consists of 10 reversible gates FG of which one gate among them has one constant input and the others have no constant input therefore $\mathrm{NCI}=1$. 
-Quantum cost: Its proposed circuit consists of $11 \mathrm{FG}$ reversible gates having $\mathrm{QC}=11$ (including $\mathrm{QC}=1$ for each) so $\mathrm{QC}=11$.

-Hardware complexity: The circuit contains 11 reversible gates of Feyman gate so $\mathrm{HC}=11(1 \alpha) \mathrm{HC}=11 \alpha$

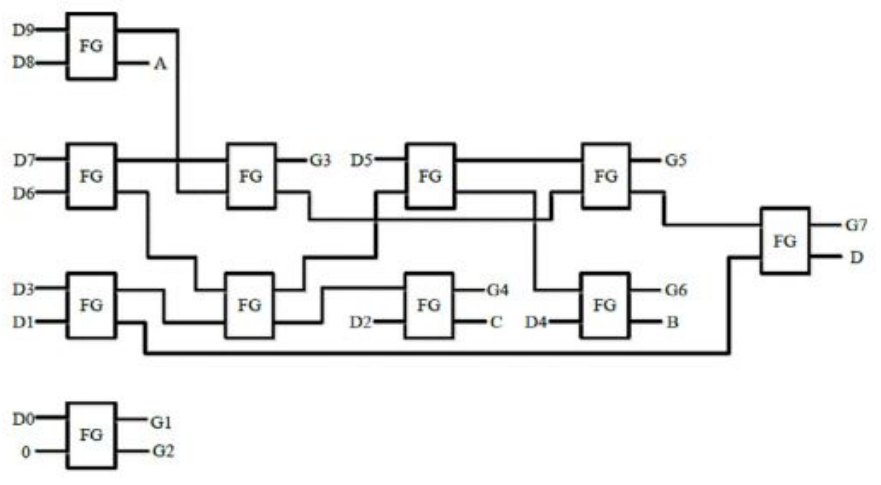

Fig. 6. Design6 D2BE.

\section{B. Design of Reversible Binary Counter with Up/Down}

It is a binary counter counting from 0 to 15 using a clock and which is linked with an up / down button that after having the impulse the counting becomes a countdown. In the following, we present the recent studies making the design of this type of binary counter.

1) Design1: IN 2016 Xuemei Q [15] proposed a design of the reversible binary counter (Fig. 7) as follows: the TFF gate is equivalent to the TFG and FG gate according to the circuit below (Fig. 8). Then based on the above we can present the performance criteria of this circuit as follows:

-Number of gates: Its proposed circuit consists of four TFF reversible gates, six gates reversible PG, three reversible gates MTG and one reversible gate FG so $\mathrm{CG}=14$.

-Number of garbage outputs: Its proposed circuit consists of three TFF reversible gates each having one garbage output, one TFF reversible gate with three garbage outputs, three reversible PG gates with one garbage output for each, three reversible PG gates with two garbage outputs for each, two reversible MTG gate with two garbage outputs for each and none of the FG reversible gates has a garbage output so NGO $=17$.

-Number of constant inputs: This circuit consists of four TFF reversible gates each having two constant inputs, six reversible PG gates having one constant input for each, three MTG reversible gates with one constant inputs for each, one reversible gate FG having one constant input so $\mathrm{NCI}=18$.

-Quantum cost: Its proposed circuit consists of four TFF reversible gates having $\mathrm{QC}=24$ (each of TFF composed of one TFG and one FG then we have QC (TFF) $=6$ because we have $\mathrm{QC}(\mathrm{TFG})=5$ and $\mathrm{QC}(\mathrm{FG})=1)$, six reversible gates PG having $\mathrm{QC}=24(\mathrm{QC}=4$ for each), three MTG reversible gates having $\mathrm{QC}=15$ ( $\mathrm{QC}=5$ for each), one reversible gate FG having $\mathrm{QC}=1$ therefore $\mathrm{QC}=64$.
-Hardware complexity: Its proposed circuit consists of four TFF reversible gates having $\mathrm{HC}=16 \alpha+4 \beta$ (each of which $\mathrm{HC}=4 \alpha+1 \beta$ because we have $\mathrm{HC}(\mathrm{TFG})=3 \alpha+1 \beta$ and $\mathrm{HC}(\mathrm{FG})=1 \alpha$ ), six reversible gates PG with $\mathrm{HC}=12 \alpha+6 \beta$ ( $\mathrm{HC}=2 \alpha+1 \beta$ for each), three reversible gates MTG having $\mathrm{HC}=3 \alpha$ (each of which $\mathrm{HC}=1 \alpha)$, and one reversible gate FG having $\mathrm{HC}=1 \alpha$ therefore $\mathrm{HC}=32 \alpha+10 \beta$.

2) Design2: In 2011 V. Rajmohan [16] proposed a design of the reversible binary counter as follows (Fig. 9):

The TFF reversible gate is equivalent to the circuit below (Fig. 10). The performance criteria of this circuit as follows:

-Number of gates: The proposed circuit consists of four gates reversible TFF (each of which is made up of two SG reversible gates and one reversible gate $\mathrm{FG}$ ), three reversible gates FRG, three reversible gates RSJ therefore $\mathrm{CG}=18$.

-Number of garbage output: Its proposed circuit consists of four TFF reversible gates, each of which has three garbage outputs (because a TFF consists of two reversible SG gates, the first gate has one garbage output and the second has two outputs and the FG gate does not have no garbage output), and the fourth gate has one more garbage output, three reversible gates RSJ each of then has one garbage output, three reversible FRG gates including two among they have one garbage output and the third has two garbage outputs so $\mathrm{NGO}=20$.

-Number of constant inputs: Its proposed circuit consists of four TFF reversible gates each having two constant inputs, six reversible PG gates having one constant input for each, three MTG reversible gates with one constant inputs for each, one gate reversible FG having one constant input so $\mathrm{NCI}=23$.

-Quantum cost: Its proposed circuit is made up of 4 reversible gates TFF having $\mathrm{QC}=44$ (because a TFF is made of two gates reversible SG and one gate $\mathrm{FG}$ then $\mathrm{QC}=11$ ), three reversible gates RSJ having $\mathrm{QC}=36$ (including each having $\mathrm{QC}=12$ ), three FRG reversible gates having $\mathrm{QC}=$ 15 (each having $\mathrm{QC}=5$ ) and a NOT gate we took it into consideration so QC $=96$. -Hardware complexity: the proposed circuit consists of four TFF reversible gates having $\mathrm{HC}=36$ $\alpha+32 \beta+8 \delta$ (because a TFF consists of two reversible SG gates having $\mathrm{HC}=8 \alpha+8 \beta+2 \delta$ and a FG gate then $\mathrm{HC}=$ $1 \alpha$ ), three reversible gates RSJ having $\mathrm{HC}=6 \alpha+3 \beta$ (each of which having $\mathrm{HC}=2 \alpha+1 \beta$ ), three FRG reversible gates having $\mathrm{HC}=6 \alpha+12 \beta+3 \delta$ (each having $\mathrm{HC}=2 \alpha+4$ $\beta+1 \delta$ ) and a NOT bear it has been taken into consideration $\mathrm{HC}=1 \delta$ so $\mathrm{HC}=48 \alpha+47 \beta+12 \delta$.

3) Design3: In 2019 Mubin Ul Haque [17] proposed a design of the reversible binary counter (Fig. 11) as following:

We can present the performance criteria of this circuit as follows:

-Number of gates: Its proposed circuit consists of four reversible MSH gates, three gates reversible HNG, and one reversible gate TS-3 and a NOT gate therefore $\mathrm{CG}=9$.

-Number of garbage outputs: Its proposed circuit consists of four MSH reversible gates each of which has two garbage outputs, three HNG reversible gates, each of which has one constant input, and one reversible gate TS-3 having no constant input so $\mathrm{NGO}=16$. 


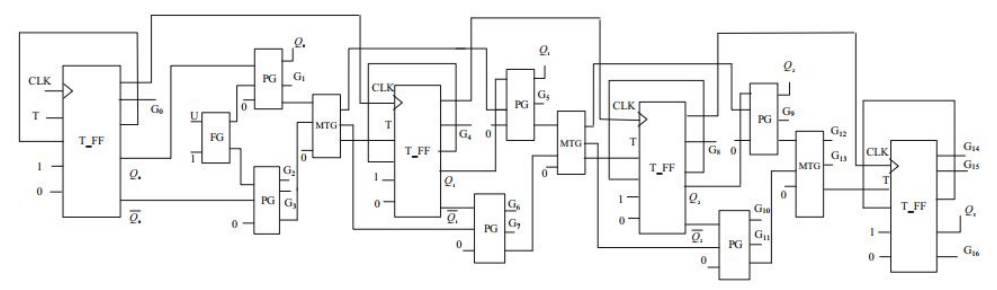

Fig. 7. Design1 Reversible 4-bit Controlled Up/Down Synchronous Counter.

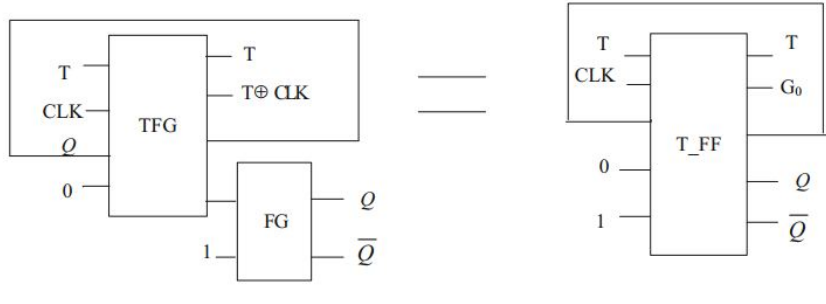

Fig. 8. Block of Reversible T Flip-flop.

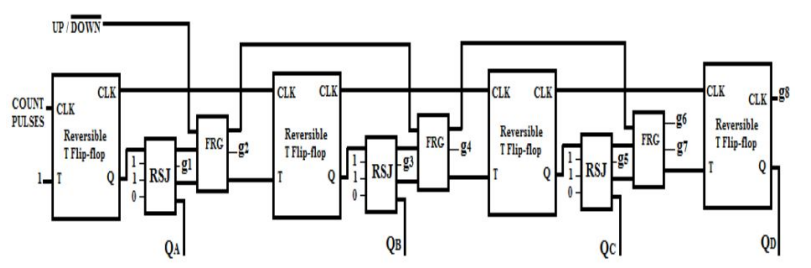

Fig. 9. Design2 Reversible 4-bit Controlled Up/Down Synchronous Counter.

-Number of constant inputs: Its proposed circuit consists of four MSH reversible gates each of which has two constant inputs, three HNG reversible gates, each of which has two garbage outputs, and one reversible TS-3 gate with two garbage outputs so $\mathrm{NCI}=7$.

-Quantum cost: Its proposed circuit consists of four MSH reversible gates having $\mathrm{QC}=24$ (of which having $\mathrm{QC}=6$ ), three reversible gates $\mathrm{HNG}$ having $\mathrm{QC}=18$ (each of which having $\mathrm{QC}=6$ ) and one reversible gate $\mathrm{TS}-3 \mathrm{QC}=2$ therefore $\mathrm{QC}=44$.

-Hardware complexity: Its proposed circuit consists of four reversible MSH gates having $\mathrm{HC}=12 \alpha+8 \beta+4 \delta$ (each having $\mathrm{HC}=3 \alpha+2 \beta+1 \delta$ ), $3 \mathrm{HNG}$ reversible gates having $\mathrm{HC}=12 \alpha+6 \beta$ (each having $\mathrm{HC}=4 \alpha+2 \beta$ ) and one reversible gate TS-3 $\mathrm{HC}=2 \alpha$ and one NOT gate having $\mathrm{HC}$ $=1 \delta$ so $\mathrm{HC}=26 \alpha+14 \beta+5 \delta$.

Limitations of previous studies: We find in these previous studies a certain limitation in terms of less optimized performance criteria, the proof is that we were able to make our designs with better performance criteria than the previous works while keeping the same functionality.

\section{Our Proposed Designs}

In this section, we will present our design of the converter from Decimal to BCD encoder, which we will be interested in obtaining the functional outputs which are:

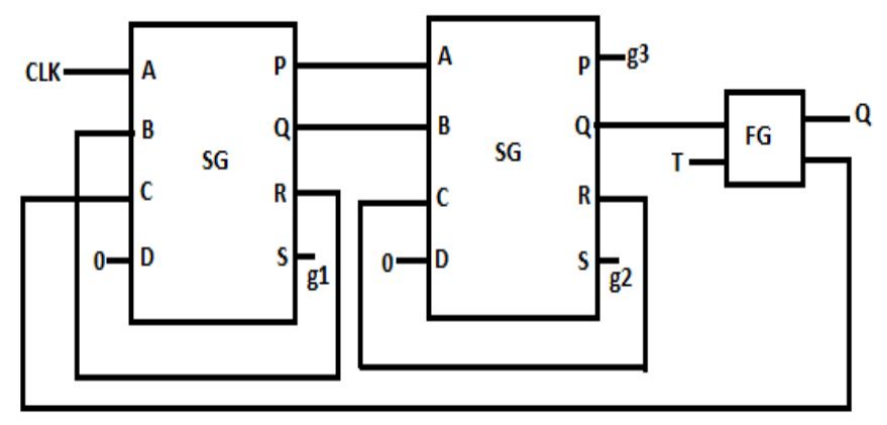

Fig. 10. Equivalent Circuit of T Flip-flop.

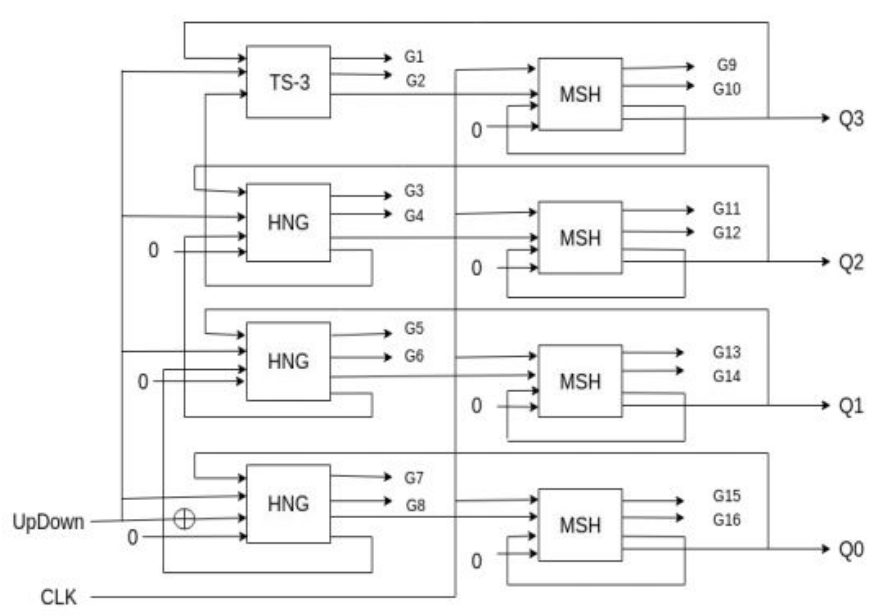

Fig. 11. Design3 Reversible 4-bit Controlled Up/Down Synchronous Counter.

$$
\mathrm{A}=\mathrm{D} 8 \oplus \mathrm{D} 9
$$

$\mathrm{B}=\mathrm{D} 4 \oplus \mathrm{D} 5 \oplus \mathrm{D} 6 \oplus \mathrm{D} 7$

$\mathrm{C}=\mathrm{D} 2 \oplus \mathrm{D} 3 \oplus \mathrm{D} 6 \oplus \mathrm{D} 7$

$\mathrm{D}=\mathrm{D} 1 \oplus \mathrm{D} 3 \oplus \mathrm{D} 5 \oplus \mathrm{D} 7 \oplus \mathrm{D} 9$

Our design is consisting of five HNFG reversible gates. Fig. 12 present our proposed circuit and its performance criteria:

* Number of gates: We have five reversible gates of the $\mathrm{HNFG}$ so $\mathrm{CG}=5$.

* Number of garbage outputs: Our design has the same functional outputs showed above. Concerning the garbage outputs, based on the functions of the reversible gates are as follows: G1 = D9, G2 = D1, G3 = D5, G4 = D9 $\bigoplus \mathrm{D} 7, \mathrm{G} 5=$ D7 $\bigoplus$ D6 Then $\mathrm{NGO}=5$.

* Number of constant inputs: We notice that there is no 
constant input therefore $\mathrm{NCI}=0$.

* Quantum cost: We have five reversible gates of the HNFG so $\mathrm{QC}=10(\mathrm{QC}=2$ for each gate $)$.

-Hardware complexity: The proposed circuit consists of five reversible $\mathrm{HNFG}$ gates having $\mathrm{HC}=10 \alpha$ (each of which having $\mathrm{HC}=2 \alpha$ ).

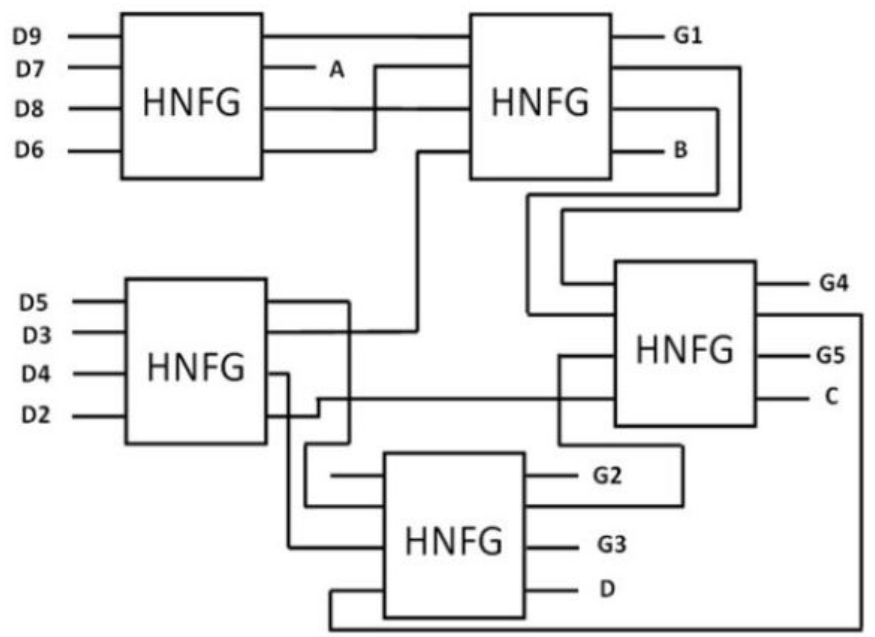

Fig. 12. Our Design of the Converter from Decimal to BCD Encoder orD2BE.

And in the other hand, we will present our proposed circuit of the reversible binary counter by showing its performance criteria while revealing the improvement percentages in terms of these parameters compared to three recent designs. Based on the circuit of [17], we replace the reversible gate MSH by the reversible gate NP since they have the same functional outputs (third and fourth output) if we fix the fourth entry by 0 , by the following, we obtain below our circuit (Fig. 13). Then based on our proposed design we can present the performance criteria of this circuit as follows:

-Number of gates: Its proposed circuit consists of four NP reversible gates, three reversible gates $\mathrm{HNG}$, and one reversible gate TS-3 and a NOT gate therefore $\mathrm{CG}=9$.

-Number of garbage outputs: The proposed circuit consists of four NP reversible gates each of which has two garbage outputs, three HNG reversible gates, each has one constant input, and one reversible gate TS-3 having no constant input so $\mathrm{NGO}=16$

-Number of constant inputs: The proposed circuit consists of four NP reversible gates each of which has two constant inputs, three HNG reversible gates, each of which has two garbage outputs, and one reversible TS-3 gate with two garbage outputs so $\mathrm{NCI}=7$.

-Quantum cost: the proposed circuit consists of four NP reversible gates having $\mathrm{QC}=20$ (of which having $\mathrm{QC}=5$ ), three reversible gates $\mathrm{HNG}$ having $\mathrm{QC}=18$ (each of which having $\mathrm{QC}=6$ ) and one reversible gate $\mathrm{TS}-3, \mathrm{QC}=2$; therefore $\mathrm{QC}=40$.

-Hardware complexity: The proposed circuit consists of four NP reversible gates having $\mathrm{HC}=12 \alpha+16 \beta+8 \delta$ (each of which having $\mathrm{HC}=3 \alpha+4 \beta+2 \delta)$, three reversible gates $\mathrm{HNG}$ having $\mathrm{HC}=12 \alpha+6 \beta$ (each of which has $\mathrm{HC}=4 \alpha+$ $2 \beta$ ) and one reversible gate TS-3 $\mathrm{HC}=2 \alpha$ and one NOT gate having $\mathrm{HC}=1 \delta$ so $\mathrm{HC}=26 \alpha+22 \beta+9 \delta$.

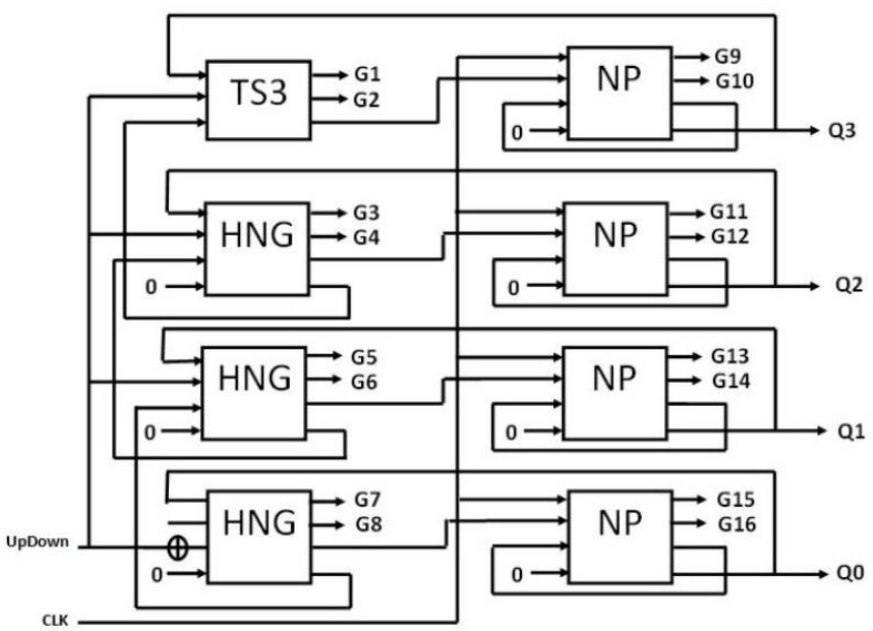

Fig. 13. Our Design of Reversible Binary Counter with Up/ Down.

\section{RESUlts AND Discussion}

So in this section, we will compare our results obtained compared to recent studies of the D2BE converter circuit and those of the reversible binary counter.

Regarding the first circuit, we will draw up a comparative table, that is, Table II showing performance criteria.

TABle II. COMPARATIVE TABle of PERFormance CRITERIA For RECENT DESIGNS OF D2BE AND OUR IMPROVEMENTS OBTAINED

\begin{tabular}{|c|c|c|c|c|c|}
\hline Circuit D2BE & CG & NGO & NCI & QC & HC \\
\hline Our design & 5 & 5 & 0 & 10 & $10 \alpha$ \\
\hline Expolited design1 [11] & 20 & 25 & - & 80 & $20 \alpha+15 \beta$ \\
\hline Expolited design2[12] & 16 & 15 & 6 & - & $20 \alpha+16 \beta+4 \delta$ \\
\hline Expolited design3[12] & 10 & 15 & 10 & 50 & $10 \alpha$ \\
\hline Exploited design4[13] & 11 & 11 & 5 & 23 & $11 \alpha+16 \beta+4 \delta$ \\
\hline Exploited design5 [14] & 10 & 15 & 9 & 40 & $20 \alpha+10 \beta$ \\
\hline Exploited design6[14] & 11 & 7 & 1 & 11 & $11 \alpha$ \\
\hline \%Imp (Design1)[11] & 75 & 80 & - & 87,5 & 50CNOT ,100AND \\
\hline \%Imp (Design2) [12] & 68,75 & 66,67 & 100 & - & 50CNOT, 100AND 100NOT \\
\hline \%Imp (Design3)[12] & 50 & 66,67 & 100 & 80 & no improvement is obtained \\
\hline \%Imp (Design4)[13] & 54,54 & 54,54 & 100 & 56,52 & 9,09 CNOT,100AND , 100NOT \\
\hline \%Imp (Design5)[14] & 50 & 66,67 & 100 & 75 & 50CNOT 100AND \\
\hline \%Imp (Design6)[14] & 54,54 & 28,57 & 100 & 9,09 & 9,09 CNOT \\
\hline
\end{tabular}

So in this section, we will compare our results obtained compared to recent studies of the D2BE converter circuit and those of the reversible binary counter.

For the design:

-Design1 [11]: 75\%, 80\%, 87.5\%,50\%, 100\% in terms of $\mathrm{CG}, \mathrm{NGO}, \mathrm{QC}, \mathrm{CNOT}, \mathrm{ND}$, respectively. -Design2 [12]: $68.75 \%, 66.67 \%, 100 \%, 50 \%, 100 \%, 100 \%$ in terms of $\mathrm{CG}$, NGO, NCI, CNOT, AND, NOT, respectively.

-Design3 [12]: $50 \%, 66.67 \%, 100 \%, 80 \%$ in terms of $\mathrm{CG}$, NGO, NCI, QC, AND, respectively. 
-Design4 [13]: 54.54\%, 54.54\%, 100\%, 56.52\%, 9,09\%, 100\%, $100 \%$ in terms of CG, NGO, NCI, QC, CNOT, AND, NOT, respectively. -Design5 [14]: 50\%, 66.67\%, 100\%, 75\% and $50 \%, 100 \%$ in terms of CG, NGO, NCI, QC, CNOT, AND, respectively.

-Design6 [14]: $54.54 \%, 28.57 \%, 100 \%, 9.09 \%, 9.09 \%$ in terms of CG, NGO, NCI, QC, CNOT Deadline, respectively.

We will present the following graph of performance criteria of D2BE designs shown in Fig. 14.

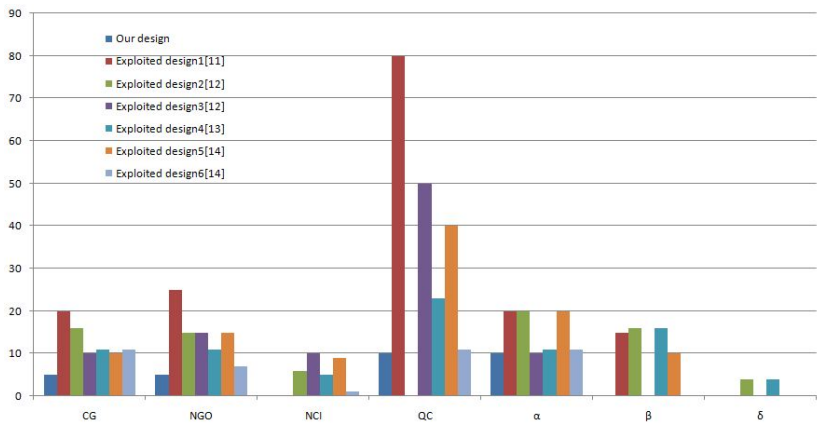

Fig. 14. Performance Criteria of D2BE Designs.

Then concerning the reversible binary counter circuit and According to the recent designs mentioned below and their performance criteria and according to our proposed design we can draw up the following comparative table (Table III).

TABle III. Comparative Table of Performance CRiteria for RECENT DESIGNS OF REVERSIBLE BINARY COUNTER WITH UP/ DOWN AND OUR IMPROVEMENTS OBTAINED

\begin{tabular}{|c|c|c|c|c|c|}
\hline Binary reversible counter & CG & NGO & NCI & QC & HC \\
\hline Our design & 9 & 16 & 7 & 40 & $26 \alpha+22 \beta+9 \delta$ \\
\hline Exploited Design 1 [15] & 14 & 17 & 18 & 64 & $32 \alpha+10 \beta$ \\
\hline Exploited Design2 [16] & 18 & 20 & 23 & 96 & $48 \alpha+47 \beta+12 \delta$ \\
\hline Exploited Design3 [17] & 9 & 16 & 7 & 44 & $26 \alpha+14 \beta+5 \delta$ \\
\hline \%Imp [15] & 35,71 & 5,88 & 61,11 & 37,5 & 18,75 CNOT \\
\hline$\%$ Imp [16] & 50 & 20 & 69,56 & 58,33 & 45,83 CNOT,53,19AND et 25 NOT \\
\hline \%Imp [17] & - & - & - & 9,09 & no improvement is obtained \\
\hline
\end{tabular}

According to this table we were able to improve the performance criteria in our proposed design compared to six recent designs as follows: For the design:

-Design1 [15]: $35.71 \%, 5.88 \%$ and $61.11 \%, 37.5 \%, 18.75 \%$ in terms of CG, NGO, NCI, QC, number of CNOT gates, respectively.

-Design2 [16]: 50\%, $20 \%$ and 69.56\%, 58.33\%, 45.83\%, $53.19 \%, 25 \% \mathrm{CG}, \mathrm{NGO}, \mathrm{NCI}, \mathrm{QC}$, number of CNOT gates, number of CNOT gates number of AND gates and number, number of NOT gates. NOT gates, respectively.

-Design3 [17]: 9.09\% in terms of QC.

According to these results obtained, they are represented in the graph below (Fig. 15).

\section{CONCLUSION}

Reversible logic occupies a significant role in reducing energy loss at the end of unused bits in the circuit compared to conventional logic computation. Our design was able to

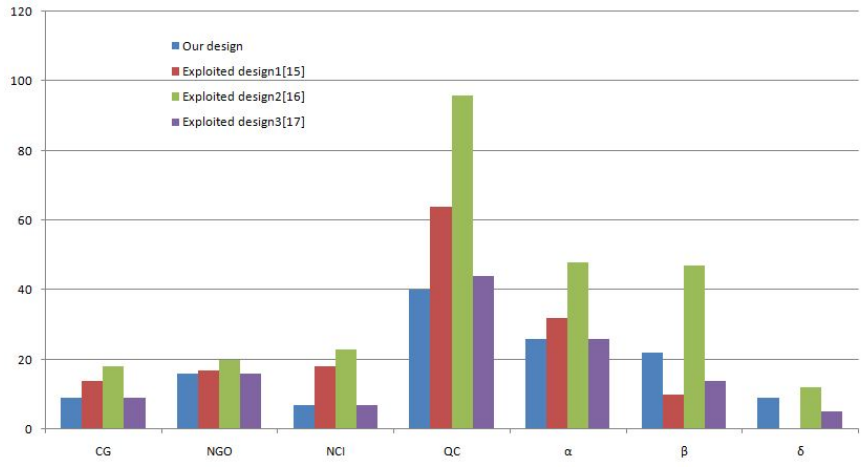

Fig. 15. Performance Criteria of Reversible 4-bit Controlled Up/Down Synchronous Counter Design.

minimize all performance criteria especially the number of garbage outputs in our D2BE circuit and the reversible binary counter,as a result a decrease in the energy dissipated at the end of unused bits because heat is directly related to fewer garbage outputs, therefore our designs are adequate for low power application. We show also an improvement in terms of other performance criteria exposing remarkable results. While waiting for new reversible gates to exploit in the future, we can optimize the D2BE circuit and that of the reversible binary counter respecting the performance, typically concerning minimizing heat energy.

\section{REFERENCES}

[1] Landauer, R.Irreversibility and heat generation in the computing process. IBM journal of research and development, 5(3), 183-191.(1961)

[2] Bennett, C. H. Logical reversibility of computation. IBM journal of Research and Development, 17(6), 525-532.(1973)

[3] Toffoli T. Reversible computing. In: de Bakker J., van Leeuwen J. (eds) Automata, Languages and Programming. ICALP 1980. Lecture Notes in Computer Science, vol 85. Springer, Berlin, Heidelberg.(1980)

[4] Perkowski, Marek \& Jozwiak, Lech \& Kerntopf, Pawel \& Mishchenko, A. \& Al-Rabadi, Anas \& Coppola, Alan \& Buller, A. \& Song, X. \& Khan, Mozammel \& Yanushkevich, Svetlana \& Smerko, V. \& Chrzanowska-jeske, M. A general decomposition for reversible logic. Proc. RM.(2001)

[5] Khlopotine, Andrei \& Perkowski, Marek \& Kerntopf, Pawel. Reversible Logic Synthesis by Iterative Compositions.. 261-266. (2002)

[6] Thapliyal, Himanshu, and M. B. Srinivas. "A novel reversible TSG gate and its application for designing reversible carry look-ahead and other adder architectures." Asia-Pacific Conference on Advances in Computer Systems Architecture. Springer, Berlin, Heidelberg, 2005.

[7] Fredkin, E., Toffoli, T. Conservative logic. Int J Theor Phys 21, 219-253 (1982).

[8] Maslov, D., \& Dueck, G. GARBAGE IN REVERSIBLE DESIGN OF MULTIPLE OUTPUT FUNCTIONS.(2003)

[9] Hung, W. N., Song, X., Yang, G., Yang, J., \& Perkowski, M.Optimal synthesis of multiple output boolean functions using a set of quantum gates by symbolic reachability analysis. IEEE transactions on Computer-Aided Design of integrated circuits and Systems, 25(9), 1652-1663.(2006)

[10] Haghparast, Majid \& Mohammadi, Majid \& Navi, Keivan \& Eshghi, Mohammad. Optimized Reversible Multiplier Circuit.. Journal of Circuits, Systems, and Computers. 18. 311-323.(2009)

[11] Wang, Junchao \& Pang, Yu \& Xia, Yang. A BCD priority encoder designed by reversible logic. 318-321.(2012) 10.1109/ICWAMTIP.2012.6413503

[12] Shukla, Vandana \& Singh, O.P. \& Mishra, G.R. \& Tiwari, Raj.A novel approach to design decimal to BCD encoder with reversible logic. 2014 
International Conference on Power, Control and Embedded Systems, ICPCES 2014.(2015) 10.1109/ICPCES.2014.7062823.

[13] Chaudhary, Kunal \& Kaur, Gurpreet.Design of Decimal to BCD Code Converter using Reversible logic and QCA. International Journal of Innovative Research in Science, Engineering and Technology. 5. 15911.(2016) 10.15680/IJIRSET.2016.0509002.

[14] Thabah, Sheba \& Saha, Prabir.New design approaches of reversible BCD encoder using Peres and Feynman gates. ICT Express. 6.(2019) 10.1016/j.icte.2019.07.001.

[15] Qi, Xuemei \& Zhu, Haihong \& Chen, Fulong \& Zhu, Junru \& Zhang, Ziyang. Novel Designs of Quantum Reversible Counters. International Journal of Theoretical Physics.(2016) 55. 10.1007/s10773-016-3122-8.

[16] Vijayan, Rajmohan \& Vijayaraghavan, Ranganathan.Design of Counters Using Reversible Logic. 5.(2011) 10.1109/ICECTECH.2011.5941973.

[17] Haque, Mubin Ul \& Sworna, Zarrin \& Afrin, Sadia \& Shihab, Fahim. A Compact Quantum Cost-Efficient Design of a Reversible Binary Counter.(2019)

[18] Surekha, M.Efficient Approaches for Designing Quantum Costs of Various Reversible Gates. International Journal of Engineering Studies. 9. 57-78.(2017) 10.37622/IJES/9.1.2017.57-78.

[19] M.A. Nielsen, I.L. Chuang, Quantum Computation and Quantum Information, Cambridge University Press, 2010.

[20] Wille, Robert \& Drechsler, Rolf. BDD-based synthesis of reversible logic for large functions. Proceedings - Design Automation Conference. 270 - 275.(2009) 10.1145/1629911.1629984.

[21] M.M. Mano, Digital Logic and Computer Design, Prentice-Hall, Inc.New York, 1979.

[22] Gaur, H. M., Singh, A. K., \& Ghanekar, U. . A review on online testability for reversible logic. Procedia Computer Science, 70, 384-391. (2015)

[23] Bhattacharya, Soham, and Anindya Sen. "A Review on Reversible Computing and it's applications on combinational circuits." International Journal 9.6 (2021).

[24] Mia, Md \& Mukib, Md \& Islam, Md. An Extended Review on Reversible Logic Gates and their Implementation. 1. 8-18.(2015) 10.5281/zenodo.3930180.

[25] Sarker, Angona \& Bahar, Ali Newaz \& Biswas, Provash \& Morshed, Monir.A Novel Presentation of Peres Gate (PG) in Quantum-Dot Cellular Automata(QCA). European Scientific Journal. 10. 101-106.(2014)

[26] Lakshmi Ranjith Kumar Kedarnath.A Novel Analysis of Sequential Circuits Design Using Reversible Logic.International Journal of Ethics in Engineering Management Education(IJEEE)(2015)

[27] RASHMI, G, Tilak B.R., Praveen. TRANSISTOR IMPLEMENTATION OF REVERSIBLE PRT GATES. International Journal of Engineering Science and Technology. 3.(2011)

[28] Haque, Mubin Ul Sworna, Zarrin Babu, Hafiz. . An Improved Design of a Reversible Fault Tolerant LUT-based FPGA. 445-450.(2016) 10.1109/VLSID.2016.39.

[29] Nowrin, Sadia Nazneen, Papiya Jamal, Lafifa. . Design of a Compact Reversible Read-Only-Memory with MOS Transistors. International Journal of VLSI Design and Communication Systems. 6. 69-84.(2015) 10.5121/vlsic.2015.6506.

[30] a n, Nagamani hv, Jayashree Bhagyalakshmi, H. . Novel low power comparator design using reversible logic gates. International Journal of Engineering Technology Sciences. 2. 566-574.(2011)

[31] Sayem, Abu Ueda, Masashi.Optimization of reversible sequential circuits. 2.(2010) 